Boise State University

ScholarWorks

Mathematics Faculty Publications and

Presentations

Department of Mathematics

7-24-2014

\title{
How Do They Know It Is a Parallelogram? Analysing Geometric Discourse at Van Hiele Level 3
}

Sasha Wang

Boise State University

Margaret Kinzel

Boise State University 


\title{
How do they know it is a parallelogram? Analysing geometric discourse at van Hiele Level 3
}

\author{
Sasha Wang and Margaret Kinzel
}

\begin{abstract}
In this article, we introduce Sfard's (2008) discursive framework and use it to investigate prospective teachers' geometric discourse in the context of quadrilaterals. In particular, we focus on describing and analysing two participants' use of mathematical words and substantiation routines related to parallelograms and their properties at Van Hiele level 3 thinking. Our findings suggest that a single van Hiele level of thinking (1959/1985) encompasses a range of complexity of reasoning and differences in discourse and thus a deeper investigation of students' mathematical thinking within assigned van Hiele levels is warranted.
\end{abstract}

Keywords: mathematical discourse; geometry; van Hiele theory

\section{Introduction}

Research in mathematical thinking has attracted increasing interest in mathematical discourse and its development. Many researchers have adopted different theoretical perspectives to shift from monolithic views of mathematical discourse and to embrace the multi-semiotic nature of mathematical activity (Moschkovich, 2010). Given our interest in students' geometric thinking, we set out to explore the characteristics of geometric discourse through a communicational approach. In particular, we report on work that combined Sfard's (2008) discursive approach with the van Hiele levels of geometric thought. By so doing, we are able to provide a more robust characterisation of students' thinking within one van Hiele level.

The Dutch educators Pierre and Dina van Hiele (1959/1985) developed a model of geometric thought, incorporating "the van Hiele levels." Many researchers (e.g., Mayberry, 1983; 1990 and Burger \& Shaughnessy, 1986) have confirmed the usefulness 
of the model for describing the development of students' geometric thinking. Central to the model is the idea that each level has its own language and symbols, that a learning process is a process of reasoning using a new mathematical language, and the levels are hierarchical. However, some researchers (e.g., Usiskin, 1982 and Crowley 1987) find the model lacking in depth and have attempted to articulate thinking processes within and across levels in more detail. Previous efforts (Hoffer, 1981 and Battista, 2009) have suggested extensions of the model through identification of skills and or reasoning at each level. The notion of learning a new language at each van Hiele level indicates the unit of analysis that viewing language as saying, doing and being (Gee, 2011) Using a discursive lens allows us to focus on what students say and do in relation to geometric figures and their properties, with the goal of providing detailed and in-depth descriptions of students' thinking processes as they are communicating mathematical ideas. In particular, our question is how the analysis of students' geometric discourse reveals the complexity of their thinking.

To address our question, we focused our analysis in two ways. First, we identified students at the same van Hiele level (level 3), and second, we chose the specific topic of parallelogram. We hypothesised that applying the discursive lens to the analysis of students' discourse at the same van Hiele level and related to a specific concept would allow us to describe similarities and differences in their thinking. Such a description contributes to the perceived need for detail within a van Hiele level. In the next section we outline both the van Hiele model and the discursive lens.

\section{Theoretical background}

The van Hiele model of thinking continues to be the best-known theoretical account of students' learning of geometric figures and their properties. The model suggests that students must progress through a sequence of discrete, qualitatively different levels of 
geometric thinking. The first four levels in the model are as follows.Level 1

(Visualisation), in which students recognise and learn the names of figures, and figures are judged by their appearance as a whole; Level 2 (Descriptive), in which students begin to recognise figures by their properties or components; Level 3 (Theoretical), where students begin to form definitions of figures based on their common properties and understand some proofs; and Level 4 (Formal Logic), in which students understand the meaning of deduction and construct mathematical proofs using propositions, axioms and theorems (van Hiele, 1986).

According to the van Hiele model, students begin to form definitions of geometric figures and to reason deductively at Level 3. However, the model does not explicitly describe thinking at this level. Battista (2007, p. 853) noted that "there is a lack of distinction between type of reasoning and qualitatively different levels in the development of reasoning" throughout the van Hiele studies. For instance, one could consider direct recognition as a type of reasoning based on intuition, and also could regard it as a period of development of geometric thinking. One challenge regarding the van Hiele model of thinking is to identify different types of reasoning and/or levels of reasoning. In their attempt, Gutierriz, Jaime and Fortuny (1991) proposed an alternative way of analysing students' geometric reasoning, and the accuracy of that reasoning, to determine the degrees of acquisition of a van Hiele level. In our work, applying a discursive lens focuses on thinking as being communicated through interaction to help differentiate different types of reasoning and shed light on how meanings are made at the same van Hiele level, as well as across different levels. In the next section, we first present Sfard's discursive framework, and then articulate our application of her framework within van Hiele level 3. 


\section{The discursive lens}

In Sfard's (2008, p. 297) communication approach to cognition, she used the term discourse as a "special type of communication made distinct by its repertoire of admissible actions and the way these actions paired with re-actions; discourse including communication in written and spoken language." In this sense, discourse goes beyond language as conventionally viewed and includes physical objects deployed for discursive ends. Sfard also made a distinction between language and discourse, viewing language as a tool, whereas discourse is an activity in which the tool is used or mediates. She proposed four characteristics of mathematics discourse: word use, routines, endorsed narratives and visual mediators. We use the specific context of parallelogram to illustrate each of these characteristics.

- Word Use (mathematical words and their use). In a geometric discourse that deals with quadrilaterals and their properties, we focus on students' use of words such as rectangle, rhombus, square, angles, sides and diagonals, to explore how meanings are communicated and made through interactions. For example, when two students are using the word rectangle, one may say it is also a parallelogram, whereas the other might disagree.

- Routines. Routines are well-defined repetitive patterns characteristic of the given discourse. For example, a student's justification routine could be to use a ruler to measure the sides of a parallelogram to show that opposite sides are congruent. Routines can be observed through conversations and analysed in terms of how students pay attention to the process of creating and justifying claims about geometric figures.

- Narratives. "A set of utterances spoken or written that is framed as a description of objects, of relations between objects, or of processes with or by objects, that 
is subject to endorsement or rejection, with the help of discourse-specific substantiation procedures" (Sfard, 2008, p. 134). In the context of geometry, endorsed narratives are evident in mathematical definitions, axioms, propositions and theorems. For example, the statement, "a parallelogram is a quadrilateral with two pairs of parallel sides" is an endorsed narrative of parallelogram, defining what a parallelogram is mathematically.

- Visual mediators. Visual mediators provide the means through which objects of geometric discourse are identified. In geometric discourse, visual mediators include the orientations and the size of geometric figures and their parts, as well as symbolic artefacts that are created specifically for written mathematical communication, such as markings to indicate parallel lines or right angles. We chose Sfard's (2008) discursive framework as an analytic tool to investigate students' geometric discourses because it provides means through which to view thought processes at the higher resolution We focused on van Hiele Level 3 as it includes at least the beginnings of deductive reasoning and formation of definitions in the context of parallelograms, to allow for a detailed analysis across students. In the remainder of this section, we articulate further our view of geometric discourse focused on parallelogram at Level 3.

Using discursive terms, geometric discourse at Level 3 includes naming a quadrilateral dependent upon on visual properties of angles and sides, as well as a common descriptive narrative (i.e., a definition) accompanying the name of the figure. That is, when a student is asked why a quadrilateral is called a "rectangle", the course of action is to check the defining conditions of rectangle by counting the number of sides (it has to be 4-sided), and by checking the conditions regarding sides (opposite sides parallel) and angles (it has right angles). So, "it is a rectangle because it has four sides, 
and it has four right angles". At this level, a student can identify that a rectangle is also a parallelogram because it fits the description of "a quadrilateral with two pairs of opposite sides parallel". For the same reason, a student begins to recognise that a square is also a rectangle, a parallelogram, and a rhombus because it fits all stated descriptions. Therefore, geometric objects at Level 3 are collections of discursive objects that begin to connect into joint categories. In the case of quadrilaterals, all 4-sided polygons begin to fall into a hierarchy of classification.

Geometric discourse at Level 3 thinking also reveals the details of substantiation of narratives as a beginning stage of deductive reasoning. Substantiation of a narrative is a discursive process of making sure that the given narrative can be endorsed or that it is true. Our discussion of students' reasoning at Level 3 focuses on two types of substantiations: an object-level and a meta-level (Sfard, 2008).

Object-level substantiation emphasises students' justification routines, looking at descriptions of how quadrilaterals are being investigated. Describing static lines, angles and polygons as movable entities under transformations (i.e., rotation, translation and reflection) as a way of substantiation is an example of object-level substantiation. Routines of substantiation that depend on measurement routines - checking sides and angles without thinking about how classes of quadrilaterals are related - are also examples at this object level. Object-level substantiation is a justification routine in which students focus on the concreteness of quadrilaterals, as figures to be transformed or measured.

Meta-level substantiation emphasises students' justification routines using endorsed narratives to endorse new narratives. That is, students use mathematical definitions and axioms to construct mathematical proofs. Students with substantiations at a meta-level may also use object-level substantiations as a type of reasoning to justify 
their conclusions. For example, a student may use the Angle-Side-Angle congruence criterion to construct a proof at a meta-level that opposite angles of a parallelogram are congruent, and could also describe why this congruence criterion works using rotations at an object-level (Sfard, 2008).

Human thinking is far more complex than our brief description may imply. We shall show that the development of geometric discourse varies from student to student at van Hiele Level 3. In particular, students' use of the word parallelogram and the ways in which they justified claims about parallelograms revealed intricacies within discourse at Level 3. We will illustrate this intricacy through an analysis of two participants' discourse related to parallelogram.

\section{Methods}

During the autumn of 2010, the van Hiele Geometry Test (Usiskin, 1982) was administered at the beginning of the semester (pretest) and ten weeks later (posttest) to sixty-three prospective primary and middle school teachers in a University in the US. Among these prospective teachers, twenty participated in two interviews, one after the pretest, and one after the posttest. Following the Cognitive Development and Achievement in Secondary School Geometry (CDASSG) project's grading method (Usiskin, 1982), each participant was assigned to a van Hiele level. When assigning a student to a level, we chose the 4 out of 5 criterion, to determine whether a participant has reached a given van Hiele level, and to minimise the chance of a participant being at that level by guessing (Usiskin, 1982). The results show that $48 \%$ of participants reached Level $3(n=30)$ at the posttest. This was anticipated as most primary and middle school geometry curricula emphasise geometric thinking up to Level 3 (Newton, 2010). Among the twenty interviewees, ten of them reached Level 3 thinking according to their van Hiele posttest. Given that half of the interviewees reached Level 3 thinking and 
given the complexity of Level 3 thinking as noted earlier, we examined their geometry discourses at Level 3.

Molly and Ivy (pseudonyms) were among the ten interviewees whose van Hiele posttest indicated their thinking reached Level 3; that is, they responded correctly to at least 4 out 5 items at each of the van Hiele Levels 1-3. The van Hiele Geometry Test provides initial information about participants' van Hiele levels at a point in time, but it does not provide rich descriptions of their geometric discourses at a particular level of thinking. Molly and Ivy are chosen to highlight the potential of the discursive lens for characterising variations within a single van Hiele level.

The interviews were designed to explore the possibilities of prospective teachers' geometric thinking through one-on-one interactions. Molly and Ivy (along with the 18 additional interviewees) participated in a 90-minute interview a week after the pretest and a 90-minute interview a week after the posttest. All interview tasks were designed to elicit participants' geometric thinking about quadrilaterals and were aligned with the van Hiele geometric test items. During the interview, all interviewees were asked to complete three tasks; however, for the purpose of this paper, we describe only the first two tasks here. The first task (shown in Figure 1), Sorting geometric figures, asked participants to place quadrilaterals into different groups. The second task (shown in Table 2), Investigating properties of parallelograms, was designed to investigate prospective teachers' understanding of the properties of parallelograms. All interviews began with the same tasks and initial interview questions; One interviewer interviewed all participants and the interviewer was required to follow an interview protocol, however, the interviews were guided based on interviewees' responses to the tasks and questions (Wang, 2011). All the interviews were video recorded. The interview 
transcripts document what participants said and also the actions they performed in the interviews.

\section{Different geometric discourse at the same van Hiele level}

Although the van Hiele posttest results showed both Molly and Ivy reached van Hiele Level 3 thinking, discursive analysis revealed differences in their geometric discourses. In the following sections, we present our analysis of Molly and Ivy's use of the word parallelogram and their substantiation routines. We compare these analyses to draw attention to the potential of the use of a discursive lens to provide additional detail within a particular van Hiele level, Level 3.

\section{Word Use}

All interviewees were presented with Task 1 and asked to sort eighteen polygons into groups. Once a grouping was completed, they were asked to justify the grouping. They were then asked to create and justify a second grouping. Subgroups could be created as needed. [Note: We refer to the eighteen polygons by their letter label.]

Molly's word use

Molly grouped the quadrilaterals into: (1) squares (U, G, and $\mathrm{R}),(2)$ rectangles $(\mathrm{M}, \mathrm{F}$, and T); (3) rhombi (Z); and (4) parallelograms (L, J, and H). She explained her grouping as follows: 

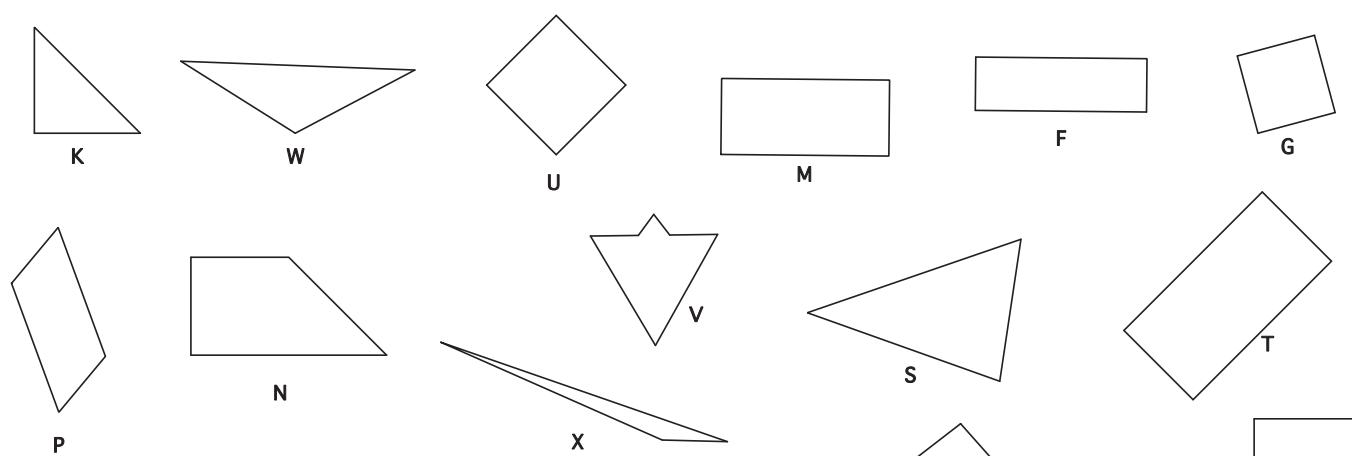

M
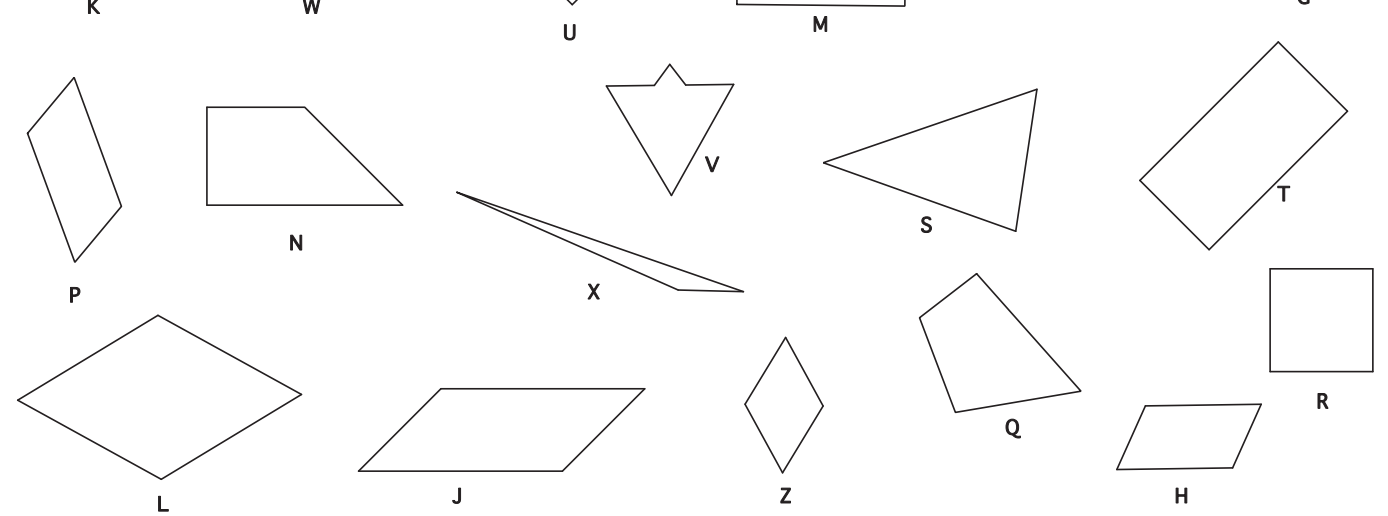

Figure 1. Sorting geometric figures

Molly: Quadrilaterals, you have your square because each form 90-degree [angles] and all side lengths are equal [Pointing at $\mathrm{U}$ ]. These are rectangles [Pointing at $\mathrm{F}$ and $\mathrm{M}$ ] because those two sides are the same. But again they form 90-degree angles.

Opposite angles are equal and opposite sides are equal, so these three would be an example of parallelogram. [Pointing to $\mathrm{L}, \mathrm{J}$ and $\mathrm{H}$ ]

Molly did not spell out exact mathematical definitions of squares, rectangles, and parallelograms, but she was able to group them together based on the common features relating to angles and sides that she observed. To investigate further, Molly was asked whether $\mathrm{J}$ and $\mathrm{Z}$, and $\mathrm{U}$ and $\mathrm{M}$, could be grouped together. She replied as follows:

Interviewer: Can I group figures $\mathrm{J}$ and $\mathrm{Z}$ together?
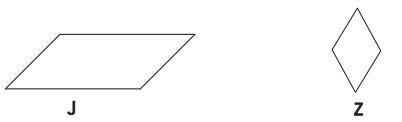

Molly: Mm Hmm [yes].

Interviewer: Why is that?

Molly: $[\ldots]$ because they both have opposite sides parallel and opposite angles are equal.

Interviewer: Can I group figures $\mathrm{U}$ and $\mathrm{M}$ together? 


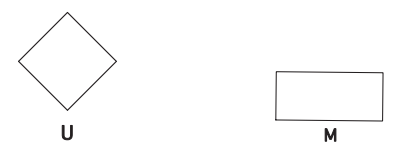

Molly: Yeah, you can because $\mathrm{U}$ has the same property as M. The only differences is that $\mathrm{M}$ does not have all the same sides length, so $\mathrm{M}$ would not have all the properties as $\mathrm{U}$, but $\mathrm{U}$ has all the properties of $\mathrm{M}$.

Molly did not initially group a rhombus and a parallelogram together, but we learned that she recognised that a rhombus is also a parallelogram. She also agreed that a square and a rectangle could be grouped together, because a square shares a property with a rectangle. In both cases, when prompted, Molly agreed that some quadrilaterals could be grouped together, but she did not on her own assign a common name (e.g., "rectangle") to the group. When Molly was asked to identify all the parallelograms among the quadrilaterals, she replied, "L, J and $\mathrm{H}$ will be just parallelograms, but all of these figures [pointing to squares, rectangles and rhombi] could be parallelograms, because they all fit to the greater property of "opposite angles and sides to be equal". It is not clear why figure $\mathrm{P}$ (another parallelogram) was missed from her grouping.

When requested, Molly provided her of definitions for square, rectangle, parallelogram and rhombus:

Table 1. Molly's definitions of parallelograms

\begin{tabular}{ll}
\hline Name & Definitions \\
\hline Square & $\begin{array}{l}\text { A Square is when all the angles form right angles and they are all the } \\
\text { same they are all } 90 \text { degrees and each side length also has to be the } \\
\text { same }\end{array}$ \\
\hline Rectangle & $\begin{array}{l}\text { A Rectangle, each angle is } 90 \text { degrees but these sides are the same } \\
\text { and parallel, and this one is the same and parallel, but not all } 4 \text { of } \\
\text { them are the same, necessarily. }\end{array}$ \\
\hline Parallelogram & $\begin{array}{l}\text { A parallelogram is when opposite sides are equal and opposite angles } \\
\text { are both equal. }\end{array}$ \\
\hline Rhombus & $\begin{array}{l}\text { As rhombus alone, it has all sides the same, but does not form 90- } \\
\text { degree angle. }\end{array}$ \\
\hline
\end{tabular}


Molly's use of the word parallelogram signified a family of quadrilaterals that share this common description: "they all fit to the greater property of opposite angles and sides to be equal". We generated the following diagram within our analysis to illustrate Molly's use of the parallelogram.

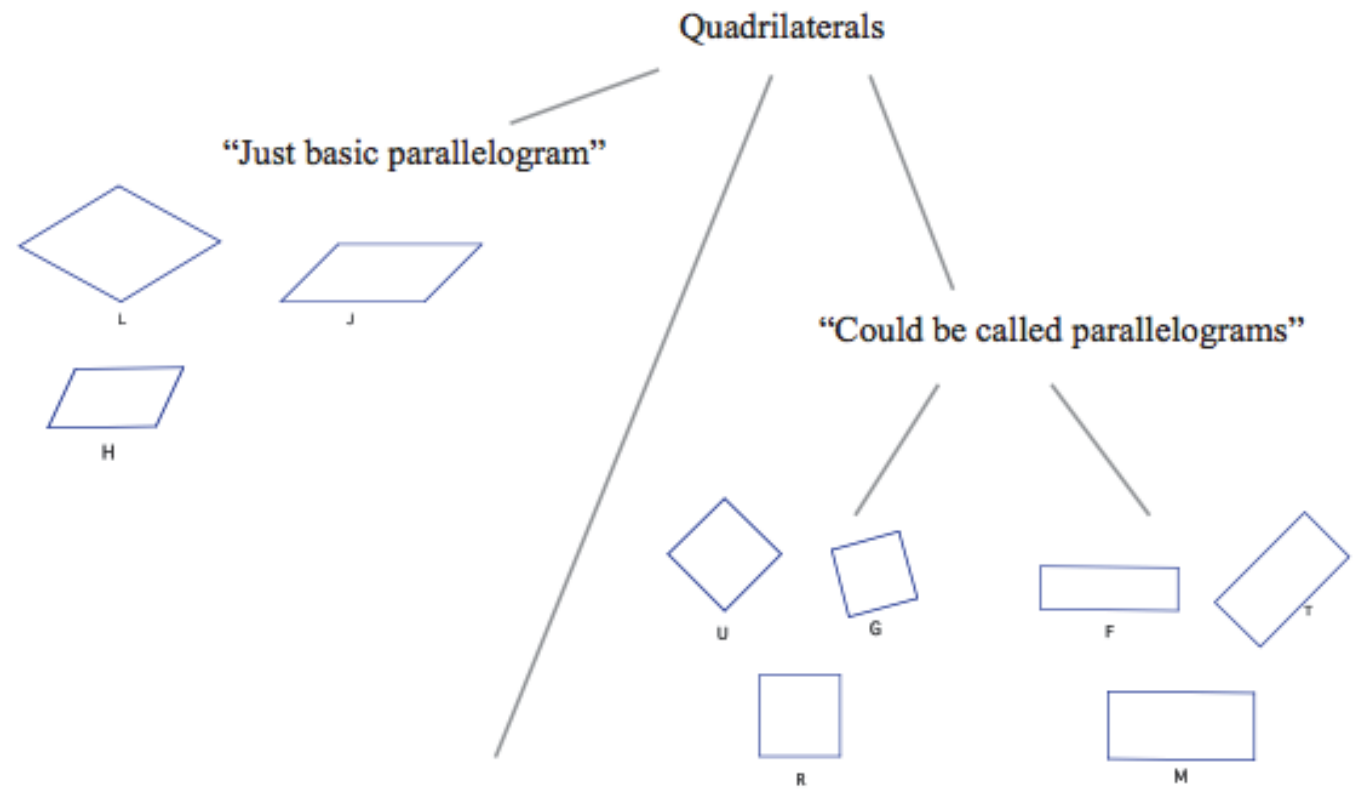

"Could be called parallelogram"

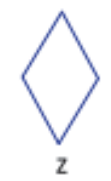

Rhombus or kite

Figure 2. An illustration of Molly's use of the word parallelogram.

When prompted, Molly agreed that these quadrilaterals--parallelograms, squares, rectangles, and a rhombus--all could be called "parallelograms", however, it appeared that she was still a bit uncomfortable accepting that they (i.e., rectangles, squares and rhombi) are "parallelograms." For example, she expressed, "as a rhombus alone, [it] does not form 90-degree angles, [but] sides are all the same", and "L, J and $\mathrm{H}$ will be just basic parallelograms." Molly's use of the word parallelogram signified prototypical shapes that are "just basic parallelograms" and those quadrilaterals that 
“could be called parallelograms". Although Molly used her definition to check if a quadrilateral is a parallelogram, from the way she communicated, her defining routines were not yet well developed at the time.

Ivy's word use

Ivy, another interviewee, was also assigned at van Hiele Level 3 thinking. However, the discursive analysis showed that Ivy's use of the word parallelogram was different than Molly's. During the post-interview, Ivy grouped all 4-sided figures $(n=13)$ into the quadrilaterals group, and included parallelograms, squares, and rectangles as parallelograms, but not all the rhombi. The rectangles group consisted of rectangles and squares, and the rhombi group included squares and rhombi. Figure 3 presents Ivy's groupings of parallelograms, rhombi and rectangles.

\section{Parallelograms group}
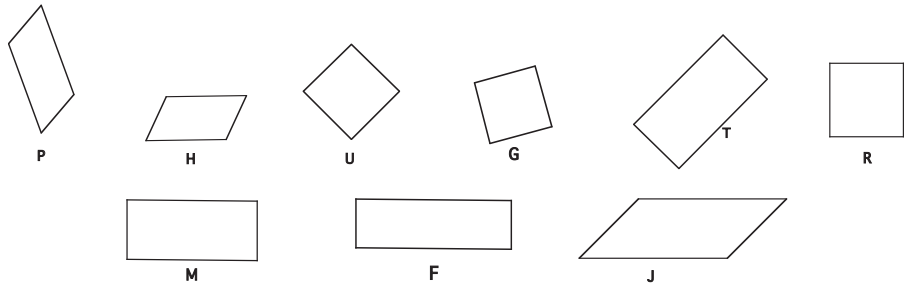

$\underline{\text { Rectangles group }}$
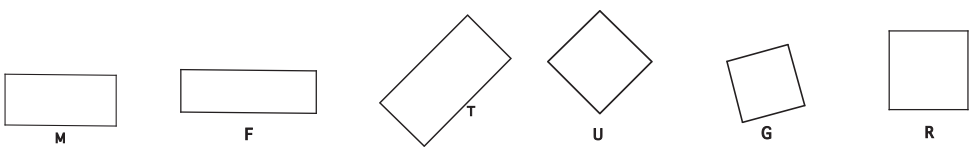

Rhombi group
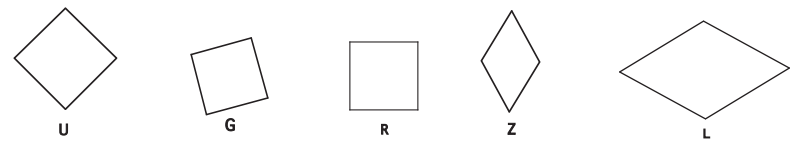

Figure 3. Ivy's grouping of parallelograms

Ivy explained, "parallelogram is two sets of opposite parallel sides, and I did the ones that are kinda obvious [pointing to J], I also did the ones that you'd think of as a rectangle and a square [pointing to $\mathrm{F}$ and $\mathrm{G}$ ] and rectangle are the ones that look like they had 90-degree angles, and I included squares cause squares are always rectangles," 
and then she continued, "rhombi have four equal sides and I have Z, R, G, U, and L

[pointing to $\mathrm{Z}$ and $\mathrm{R}$ ]", and verified that both squares and rhombi were rhombi because

"that is what a rhombus is, four sides of equal length". To justify her responses in

grouping rectangles and parallelograms together, Ivy also argued, “they're

parallelograms because they have two sets of opposite sides parallel".

Ivy used a definition of parallelogram to group all qualified quadrilaterals, and classified the quadrilaterals beginning with the attributes of their sides. When asked to create a different grouping, Ivy split her parallelograms group into parallelograms and rectangles, and split the rhombus group into rhombi and squares by the characteristics of right angles. We generated the following diagram to illustrate Ivy's use of the parallelogram.

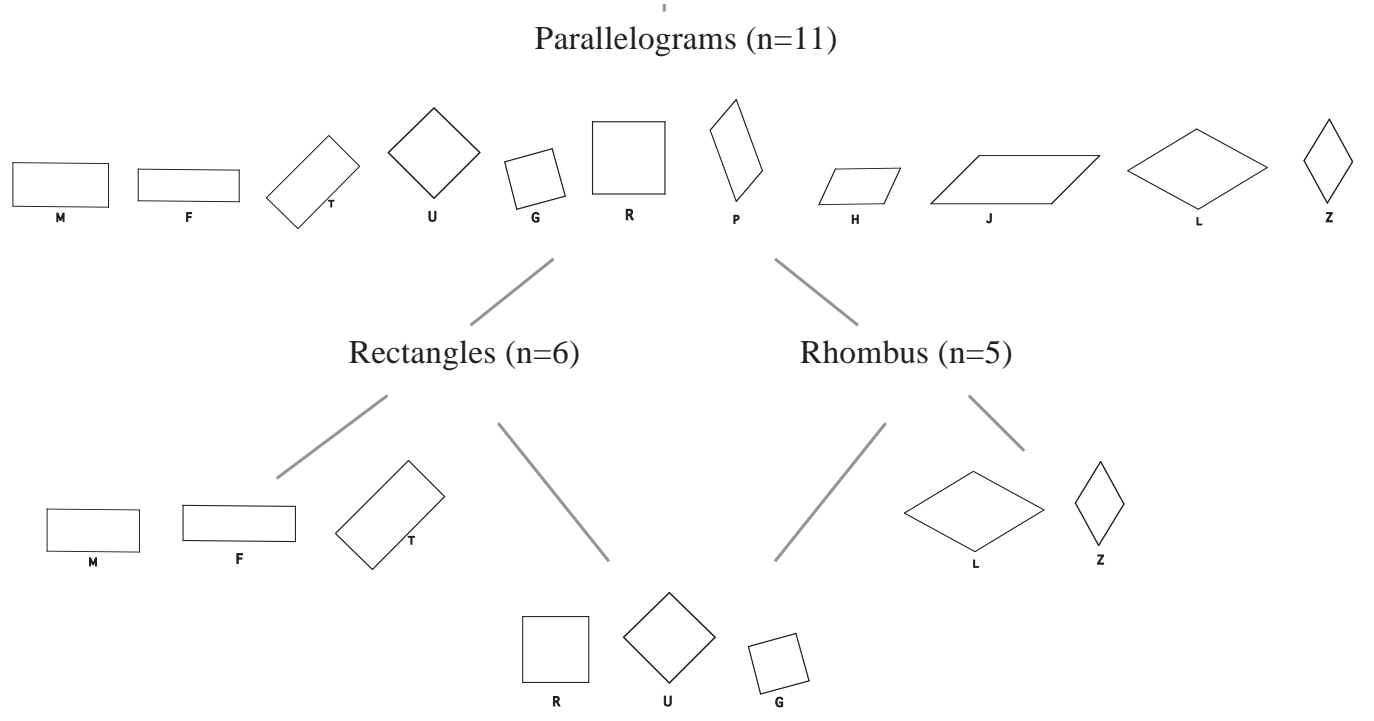

Figure 4. An illustration of Ivy's use of the word parallelogram.

In Ivy's case, her use of the word parallelogram signified a more hierarchical (and nested) classification of quadrilaterals. To Ivy, these parallelograms may have different visual appearances, some have right angles and some do not, but they all share a common descriptive narrative, "opposite sides parallel and equal". That is, Ivy focused on using a definition of parallelogram to identify a collection of quadrilaterals. 
Both Molly and Ivy applied definitions in identifying parallelograms. However, Molly represents the case of a student who is still developing the use of definitions at Level 3, whereas Ivy represents a case of a student who gave a definition of parallelograms at that Level.

\section{Substantiation Routines}

In the following section, we examine another characteristic of Molly and Ivy's geometric discourse, substantiation routines. During the post-interview, they were presented with Task 2, Investigating the properties of parallelograms.

Table 2. Investigating the properties of parallelograms (Task 2).

Draw a parallelogram in the space below.

What can you say about the angles of this parallelogram?

- What can you say about the sides of this parallelogram?

o What can you say about the diagonals of this parallelogram?

Draw a new parallelogram that is different from the one you drew previously.

○ What can you say about the angles of this parallelogram?

- What can you say about the sides of this parallelogram?

- What can you say about the diagonals of this parallelogram?

\section{Molly's substantiation routines}

Molly's work on Task 2 included her drawings of a parallelogram and a rectangle. She drew a parallelogram first and then a rectangle when asked to produce a different example. When the interviewer asked her about the properties of the parallelogram, she responded,

Interviewer: What can you say about the sides of this parallelogram? 
Molly: Opposite sides are equal and they should be equal.

Interviewer: Why do you say 'they should be'?

Molly: Because it has the properties of a parallelogram. By looking at it, it looks as if they are, so it could be good.

Interviewer: Can show me why the opposite sides are equal and parallel?

Molly: Based on the property of it [the parallelogram].

At this point, Molly's course of actions consisted of visual recognition "by looking at it" and visually checking the sides, to verify her claim of opposite sides were parallel and equal. She used this narrative "the properties of it [parallelogram]" to confirm her claim. Molly provided a similar response when she discussed the diagonals of the parallelogram.

Interviewer: What can you say about the diagonals of the parallelogram?

Molly: They bisect whatever angles these are.

Interviewer: How do you know diagonals bisect angles?

Molly: It's the properties of it [parallelogram].

Molly's claim that diagonals of this parallelogram (the one she drew) bisect the angles is not correct. She used the same narrative "it's the property of it" to confirm her claim.

To continue Task 2, Molly drew a rectangle as a new parallelogram that was different than the first one (a parallelogram). When asked about the properties of the rectangle, Molly's response of the diagonals of this parallelogram (a rectangle) is as follows:

Interviewer: What can you say about the diagonals of this parallelogram?

Molly: The diagonals would have to be equal, form these right triangles, specifically 90-degree. [Draw diagonals] 


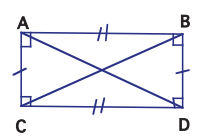

Molly: it takes half of these, so it'd be 45, 45, 90 .

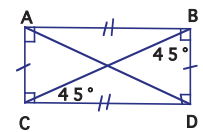

Molly: Two diagonals form two congruent triangles because they have the same

base lengths, side lengths. [Referring to shaded and un-shaded triangles]

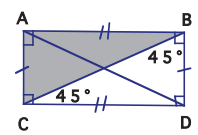

Molly: [Diagonals] bisect the angles.

Interviewer: How do you know they [diagonals] bisect angles?

Molly: This is one of our right triangles. [Shade the triangle].

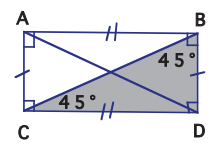

Molly: This is 90-degree angle here. [Pointing at the $\angle \mathrm{D}$ ]

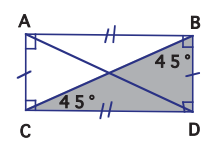

Molly: This diagonal [referring to the hypotenuse of the shaded triangle]

completely bisects these two angles $(\angle \mathrm{B}$ and $\angle \mathrm{C})$ in half because we have our 45-, 45-90-degree angles. You know that the [angles of] triangle has to be equal to180.

Again, Molly's claim about diagonals bisecting angles in a rectangle is incorrect.

Molly's substantiation routines of "diagonals bisect the angles" consisted only of recalling and visual recognition in identifying partial properties of a parallelogram. In discursive terms, recalling is a routine that one performs to summon a narrative that was endorsed in the past, or to recall narratives that were memorised in the first place (Sfard, 2008). Because Molly was confident about her conclusions of "diagonals bisect the angles" in a parallelogram and a rectangle, no further verifications were needed. Molly demonstrated incorrect deductive reasoning when she talked about the properties of 
parallelograms, however she did use deductive reasoning, not explicitly, to verify the “diagonals form two congruent triangles". Molly's substantiation routines were selfevident, and so were neither at an object-level (concrete comparison or measurement) nor at a meta-level (deductive logic).

Ivy's substantiation routines

Ivy's work on Task 2 included drawings of a parallelogram and a square. Her substantiation routines involved informal deductive reasoning. Ivy's written responses to the question, "what can you say about the angles of this parallelogram?" are shown in Figure 5.

\section{A. Draw a parallelogram in the space below.}

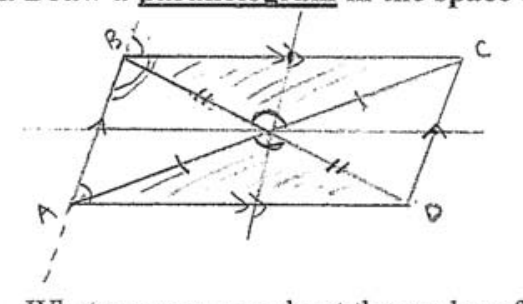

1. What can you say about the angles of this parallelogram?

$$
\begin{array}{lll}
\angle A=\angle C & \angle A+\angle B=180^{\circ} & \angle C+\angle B=180^{\circ} \\
\angle B=\angle D & \angle D+\angle C=180^{\circ} & \angle D+\angle A=180^{\circ}
\end{array}
$$

Figure 5. Ivy's written responses

Ivy drew a parallelogram by constructing a quadrilateral with opposite angles equal, as she stated, "this is a parallelogram because I drew it, so angle A is equal to angle $\mathrm{C}$ and angle $\mathrm{B}$ is equal to angle D." When talking about the angles of the parallelogram, Ivy also stated that the two consecutive angles of the parallelogram add up to $180^{\circ}$. Her justification of $\mathrm{m} \angle \mathrm{A}+\mathrm{m} \angle \mathrm{B}=180^{\circ}$ is as follows:

Ivy: If you were to extend this line $[$ Side $\mathrm{AB}] \ldots$

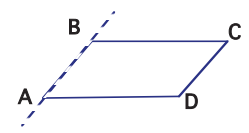

Ivy: You could look either way, like this angle is equal to this angle. $\mathrm{BC}$ and $\mathrm{AD}$ are parallel. They [pointing at the marked angles] are corresponding angles 
because they are on the parallel lines.

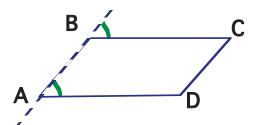

Ivy: Then you could tell that if you add these two angles, it's angles on a line

[Pointing at $\angle \mathrm{B}$ and its exterior angle]. So it's 180 degrees.

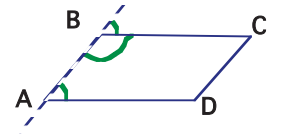

Ivy: So angle A and angle B add up to 180 degrees.

To verify $\mathrm{m} \angle \mathrm{A}+\mathrm{m} \angle \mathrm{B}=180^{\circ}$, Ivy extended side $\mathrm{AB}$ so that the structure of the corresponding angles formed by parallel lines and their transversals was visible. She stated that the corresponding angles were equal because they were on the parallel lines, and then concluded that angle A and angle B add up to 180 degrees. Although Ivy verified her claim informally, it is important to note that she justified her claim using an endorsed narrative, sides $\mathrm{BC}$ and $\mathrm{AD}$ are parallel, knowing that quadrilateral $\mathrm{ABCD}$ is a parallelogram.

Ivy also talked about the diagonals of the parallelogram. She stated, "the diagonals of a parallelogram bisect each other", and added, "they [the diagonals] create corresponding triangles".

Ivy: They [the diagonals] cross at one point.

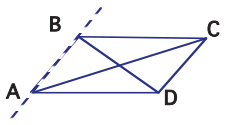

Ivy: They create corresponding triangles. Well, like this triangle corresponds with this triangle [Shaded the two corresponding triangles]

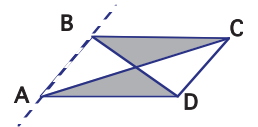

Interviewer: What do you mean by "corresponding triangles"?

Ivy: This angle and this angle are equal, cause they're vertical angles...

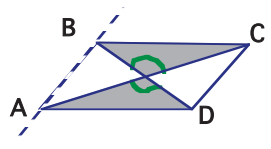


Ivy: And then, this side should equal this side...

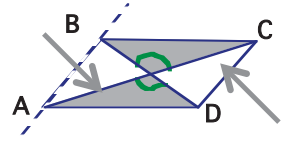

Ivy: And this side should equal this side. And I know they're corresponding through Side-Angle-Side. Like, that would be the rule that...

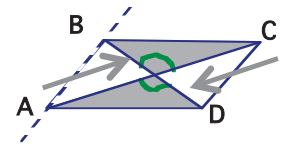

Interviewer: How do you know these sides are equal?

Ivy: Because diagonals bisect each other.

Interviewer: How do you know they [diagonals] bisect each other?

Ivy: I don't really know how I know... I guess it's because the sides are equal length and they're parallel.

In the preceding interaction, Ivy started with a descriptive narrative about the diagonals of a parallelogram, "they cross at one point," and then asserted that the diagonals created corresponding triangles. She then verified the corresponding triangles were a pair of [congruent] triangles with the Side-Angle-Side (SAS) criterion. Here, Ivy used an endorsed narrative "diagonals bisect each other" to show that the corresponding triangles [were congruent]. However, when asked how she knew the diagonals of this parallelogram bisect each other, Ivy responded, “I don't really know...I guess, it's because the sides are equal length and they're parallel."

Ivy did not just use the SAS criterion to verify congruent triangles, she also identified corresponding triangles, and the three elements needed for verification of congruent triangles. She recalled, "diagonals bisect each other" as a fact to justify the congruency of the sides, and used vertical angles to show the congruence of included angles. In this case, Ivy used an endorsed narrative "diagonals bisect each other" to verify her claim, but she did not know why the narrative was true. 
To complete Task 2, Ivy drew a square as a new parallelogram. When asked about the diagonals of the square, Ivy provided two narratives, namely, "they're equal", and "they bisect the angles." She applied her knowledge of the diagonals in a rectangle, "the same way I knew with the rectangle" to the case of a square. Ivy's routines of justifying the statement "the diagonals bisect the angles" were analysed. As an illustration, we summarise Ivy's substantiation routines, with corresponding transcripts. Table 3. An illustration of Ivy's substantiation routine.

\section{Routine}

1. Declare narratives

1.1Draw a diagonal

1.3 Identify the relation between the angles and sides of the right triangle.

\section{Verification of} isosceles triangle 2.1 Identify congruent sides of the triangle

\subsection{Verification of congruent angles} 2.3 Finding the angle measures of $\mathrm{X}$ and $\mathrm{Y}$

2.4 Finding other angles measures

\section{Transcripts}

Diagonals bisect the angles

I guess I'd draw a diagonal

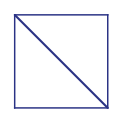

It splits the square into two right triangles, because all of these angles are 90-degrees.<smiles>C1C2C3C1C23</smiles>

\section{Adding right angle signs on all angles}

By the angle sum rule, all angles add up to 180 degrees [in a triangle]. You already have 90 here. So, X plus Y has to equal 90. It's also an isosceles triangle.

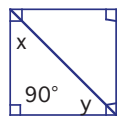

Assigning $X$ and $Y$ to the two angles

Interviewer: How do you know it's an isosceles triangle?

These two sides equal.<smiles>C1C2CC3C1C23</smiles>

Adding two marks on the sides

It's an isosceles triangle. So $\mathrm{X}$ is equal $\mathrm{Y}$.

I know that $\mathrm{X}$ and $\mathrm{Y}$ have to equal 90 degrees. So, I know that $\mathrm{X}$ is 45 degrees and $\mathrm{Y}$ is 45 degrees.

So, if you know it's 90, and $\mathrm{Y}$ is equal 45 degrees, and this angle is also 45 degrees. Same for $\mathrm{X}$ here. So diagonals splitting into two equal angles and they are 45 degrees each.

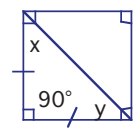

"this angle is also 45 degrees" 
3. Conclusion Yeah, diagonals bisect the angles.

We saw that Ivy favoured algebraic reasoning in her substantiation routines. She labelled the angles $\mathrm{X}$ and $\mathrm{Y}$, and used an endorsed narrative, "all angles are 90 degrees" to justify that $\mathrm{X}$ and $\mathrm{Y}$ were the angles of a right triangle. She used another endorsed narrative, "these two sides equal" to verify that the triangle was isosceles. Finally, Ivy solved for $\mathrm{X}$ and $\mathrm{Y}$ algebraically, to find that they were 45 degrees each. Using this newly endorsed narrative, Ivy concluded that the diagonals bisect the angles. In this example, Ivy's substantiation routines are a mixture of algebraic and informal deductive reasoning.

Although the van Hiele geometry test indicated that both Molly and Ivy were at van Hiele Level 3 (theoretical) thinking, our interview analysis reveals significant differences in their geometric discourses. These differences were not immediately detected, but were from Molly and Ivy's responses to the tasks and different interview probing based on their responses. For example, Molly grouped quadrilaterals into groups of squares, rectangles, rhombi and parallelograms, and based on this response, the interviewer probed the questions of "Can I group a parallelogram and a rhombus together?" and "Can I group a square and a rectangle together?" to elicit her thinking of the relationships between different parallelograms. As we noted earlier, Molly did not initially group a rhombus and a parallelogram, a rectangle and a square together, but she recognised that a rhombus is also a parallelogram, and a square is also a rectangle after these interview probing. By contrast, Ivy first grouped quadrilaterals into groups of rectangles (with squares), parallelograms (with squares and rectangles) and rhombi (with squares), and based on this response, Ivy was asked to regroup the quadrilateral differently to confirm her understanding of the relationships between different parallelograms. The interviewer probed different questions based on interviewee's 
responses to the tasks, to bring out their thinking about the relationships between the parallelograms, and to make their thinking more explicit to others. Using discursive terms, Figures 6 and 7 illustrates the characterizations of word use and substantiation routines in the context of parallelogram. In each figure, the left branch refers to the participant's word use and the right branch to her substantiation routines.

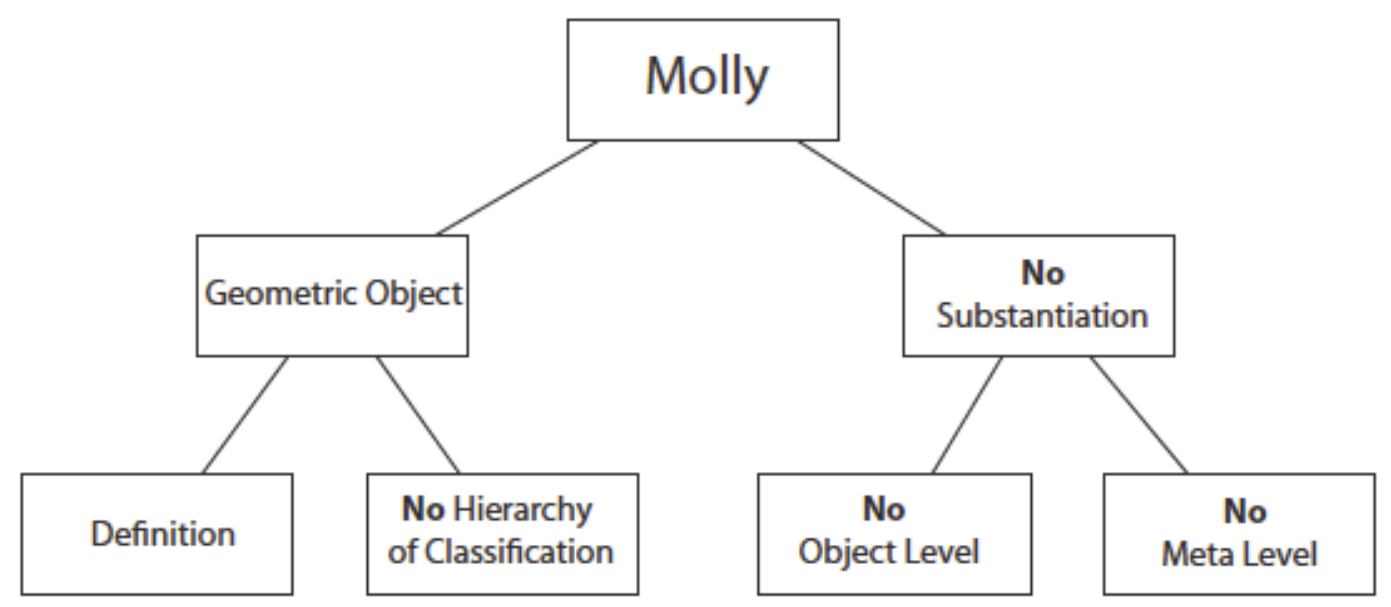

Figure 6. An illustration of Molly's geometric discourse

At van Hiele Level 3, Molly had developed some knowledge about quadrilaterals and was able to use her definitions to identify parallelograms. However, parallelograms were not clearly connected in a hierarchy of classifications. Further, Molly showed the ability to use deductive reasoning at the beginning stage of Level 3. Her substantiation routines appear to be self-evident, not requiring justifications, neither at an object-level nor a meta-level. 


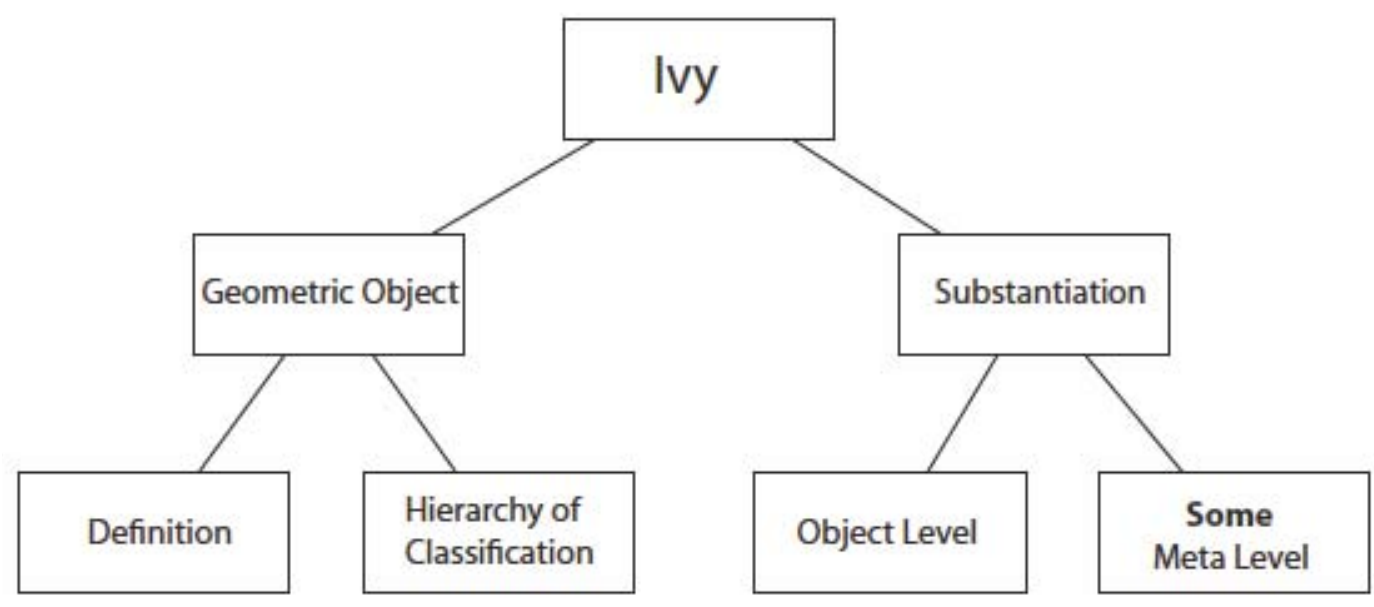

Figure 7. An illustration of Ivy's geometric discourse

By contrast, Ivy exhibited different characteristics in her geometric discourse.

Ivy identified quadrilaterals by definitions and demonstrated an understanding of the hierarchical of classification of parallelograms. She used informal deductive reasoning, employing geometrical propositions to verify claims. In addition, she also applied algebraic reasoning to further justify her claims at a meta-level.

\section{Conclusion}

Revisiting van Hiele level 3 with a discursive lens provides details of what students mean when they say, "it is a parallelogram", by analysing the words they use and the discourse-specific substantiation routines they engage.

There are differences in geometric discourses at van Hiele Level 3. With respect to the word parallelogram and its use, we observed that it had different meanings to students depending on how they defined it. We found that Molly's knowledge of definitions for quadrilaterals was not well connected, whereas Ivy used her definitions to demonstrate her understanding of parallelograms through a hierarchical classification. In terms of substantiation routines, we identified their ability to construct mathematical proofs at different stages. For example, Ivy used endorsed narratives to 
substantiate her claims and she constructed informal proofs using congruence (i.e., Side-Angle-Side) criterion to endorse new narratives. By contrast, Molly also used deductive reasoning to show the diagonals of a parallelogram form two congruent triangles in order to endorse a narrative of "diagonals of a parallelogram would be equal", but her substantiation was incomplete. When asked to justify her claims of “diagonals bisect the angles in a rectangle and a parallelogram”, Molly's courses of actions were self-evident and therefore the substantiation routines were absent. Both participants reached Level 3 thinking, but their geometric discourses were quite different regarding the word use and substantiation routines.

In looking at the details of the discursive analysis, we distinguish two very important features of van Hiele level 3 thinking. The first is the ability to use definitions within a hierarchy, a feature that characterises the student's knowledge of mathematical definitions; and second is the ability to construct informal proofs, a salient feature that characterises the student's beginning stage of deductive reasoning skills. The process of developing Level 3 thinking entails both using definitions fluently and learning to construct mathematical proofs. In addition, Molly and Ivy's geometric discourses demonstrate that the degrees of acquisition of a van Hiele level can vary among individual students (Gutierriz, Jaime \& Fortuny,1991). One might question whether Molly's geometric thinking had in fact reached Level 3, our analysis of her geometric discourse indicated that she had a low acquisition of Level 3 because her knowledge of definitions and abilities of reason deductively were not well developed.

Examining students' geometric discourses at Level 3 also raised questions about the relations between students' use of definitions and their ability to construct informal proofs. When a student (like Molly) is still developing her knowledge of definitions, it might delay the development of routines of substantiation; in Molly's case, these 
substantiation routines were absent. When a student (like Ivy) is able to construct informal proofs, her defining routines might have helped to develop the routines of substantiation; that is, Ivy's understanding of definitions may have supported her ability to construct informal proofs. Discursive analysis poses a new question: how do the two features of van Hiele level 3 interact as students develop an understanding of proofs?

Approaching the van Hiele theory with a discursive perspective, our analysis suggests that a single assignment of van Hiele level does not encompass the range of complexity and variation of geometric thinking among individual students. This demonstrates the benefits of revisiting the van Hiele model of thinking with multiple lenses in order to better understand how geometric thinking develops.

\section{References}

Battista, M.T. (2007). The development of geometric and spatial thinking. In F. K.

Lester (Ed.), Second Handbook of Research on Mathematics Teaching and Learning

(Vol. 2, pp. 483-908). Charlotte, NC: Information Age Publishing.

Battista, M.T. (2009). Highlights of research on learning school geometry. In T.V.

Craine \& R. Rubenstein (Eds.), Understanding geometry for a changing world

(pp.91-108). Reston, VA: National Council of Teachers of Mathematics.

Burger, W. F., \& Shaughnessy, J. M. (1986). Characterizing the van Hiele levels of development in geometry. Journal for Research in Mathematics Education, 17(1), $31-48$. 
Crowley, M. L. (1987). The van Hiele model of the development of geometric thought.

In M. Lindquist (Ed.), Learning and teaching geometry, K-12 (pp. 1-16). Reston, VA: National Council of Teachers of Mathematics.

Crowley, M. L. (1990). Criterion referenced reliability indices associated with the van Hiele geometry test. Journal for Research in Mathematics Education, 21, 238-241.

Gee, J. (2011). An introduction to discourse analysis: theory and method.

Gutierrez, A., Jaime, A., \& Fortuny, J. M. (1991). An alternative paradigm to evaluate the acquisition of the van Hiele levels. Journal for Research in Mathematics Education, 22, 237-251.

Hoffer, A. (1981). Geometry is more than proof. Mathematics Teacher,74, 11-18.

Mayberry, J. (1983). The van Hiele levels of geometric thought in undergraduate preservice teachers. Journal for Research in Mathematics Education, 14(1), 58-69

Moschkovich, J. N. (2010) Recommendations for research on language and mathematics education. Moschkovich, J. N. (Ed.). Language and mathematics education: Multiple perspectives and directions for research (pp.151-170). Information Age Publishing.

Newton, J. (2010). An examination of K-8 geometry state standards through the lens of the van Hiele levels of geometry thinking. Smith, J. P III (Ed.). Variability is the rule: A companion analysis of $K-8$ state mathematics standards. Information Age Publishing.

Sfard, A. (2008). Thinking as communicating: human development, the growth of discourses, and mathematizing: Cambridge. 
Usiskin, Z. (1982). Van Hiele levels and achievement in secondary school geometry

Chicago: University of Chicago.

Van Hiele, P. M. (1959/1985). The child's thought and geometry. In D. Fuys, D. Geddes

\& R. Tischler (Eds.), English translation of selected writings of Dina van Hiele-

Geldof and Pierre M. Van Hiele (pp. 243-252). Brooklyn, NY: Brooklyn College, School of Education.

Van Hiele, P.M. (1986). Structure and insight: A theory of mathematics education.

Orlando, FL: Academic Press.

Wang, S. (2011). The van Hiele theory through the discursive lens: Prospective teachers' geometric discourses (Doctoral dissertation, Michigan State University). Available from ProQuest Dissertations and Thesis database. (UMI No. 3489785). 